\title{
TOWARDS A NEW SDN NFV APPROACH FOR THE MANAGEMENT OF MPLS INFRASTRUCTURES
}

Faycal Bensalah

Ph.D Student, Network and Telecommunications team.

Faculty of Sciences, Chouaib Doukkali University.

El Jadida (Morocco).

E-mail: f.bensalah@ucd.ac.ma

Najib E1 Kamoun

Ph.D, professor in the dept. of physics.

Researcher member on STIC laboratory, header of Network and Telecommunications team. Faculty of Sciences, Chouaib Doukkali University.

El Jadida (Morocco). E-mail: elkamoun@ucd.ac.ma

Recepción: 29/07/2019 Aceptación: 19/09/2019 Publicación: 06/11/2019

\section{Gitación sugerida:}

Bensalah, F. y El Kamoun, N. (2019). Towards a new SDN NFV approach for the management of MPLS infrastructures. 3C Tecnología. Glosas de innovación aplicadas a la pyme. Edición Especial, Noviembre 2019, 107-1 19. doi: http://dx.doi.org/10.17993/3ctecno.2019. specialissue3.107-119

\section{Suggested citation:}

Bensalah, F. \& El Kamoun, N. (2019). Towards a new SDN NFV approach for the management of MPLS infrastructures. 3 C Tecnología. Glosas de innovación aplicadas a la pyme. Speciaal Issue, November 2019, 107-119. doi: http://dx.doi.org/10.17993/3ctecno.2019. specialissue3.107-119 


\section{ABSTRACT}

Network infrastructure virtualization has become an active research area. Indeed, network feature virtualization (NFV) brings unparalleled flexibility to nextgeneration networks and goes far beyond cost reduction. This technology reduces vendor dependency and allows new features to be deployed faster than ever before. In this article we propose a new solution for the virtualization of network services, particularly in relation to concepts related to MPLS technology. Our solution ensures fast access to the access network while guaranteeing quality of service.

\section{KEYWORDS}

NFV, SDN, MPLS, Automation. 


\section{INTRODUCTION}

NFV (virtualization of network functions) is a way to reduce costs and accelerate service deployment for network operators by dissociating functions such as firewalls or encryption from any dedicated hardware and moving them to virtual servers (Hawilo, Shami, Mirahmadi, \& Asal, 2014). The NFV concept for virtualization of network functions is like a new step towards creating a more agile and cost-effective network infrastructure. Network function virtualization (NFV) extracts network functions, allowing software components running on standardized compute nodes to install, control and manipulate them. NFV integrates cloud and virtualization technologies to quickly develop new network services while optimizing flexibility in terms of scalability and automation. These technologies are often combined in NFV and SDN solutions. This virtualization of network functions reduces network operators' dependence on dedicated hardware and improves scalability and customization across the entire network. Unlike a virtual network, the NFV only seeks to offload certain network functions rather than the entire network.

The NFV reduces the need for dedicated hardware to deploy and manage networks by transferring network functions to software that runs on standard hardware and can be managed from anywhere on the operator's network.

The separation of network functions from hardware provides many advantages for the network operator, including:

- Reduction of the space required for the network's physical equipment.

- Reduction of grid power consumption.

- Reduction of network maintenance costs.

- Simplification of network upgrades.

- Extension of the life cycle of network physical equipment.

- Reduced maintenance and material costs. 
The desire to automate the orchestration and management of the network, storage and computing resources is a key factor in NFV and SDN development. Imagine a scenario involving a physical server with 10 virtual machines or hundreds of containers. This concept cannot be evolutionary if it involves manual operations. Automation enables virtualized network functions (VNFs) such as virtual machines (VMs), containers, routers, firewalls and intrusion prevention systems (IPSs) to be quickly created or removed to automatically adapt them to dynamic demand.

SDN and NFV are not an integral part of each other (Bahnasse, Louhab, Oulahyane, Talea, \& Bakali, 2018). While the two concepts are quite different, they are nevertheless related. The NFV can thus be implemented without the need for an SDN, although these two approaches can be combined to provide greater added value.

The objectives of the NFV can be achieved by using non-SDN mechanisms, using techniques currently used in many datacenters. But approaches based on the separation of control and data transfer plans, as proposed by the SDN, can improve performance, simplify compatibility with existing deployments and facilitate operation and maintenance procedures.

The NFV can support the SDN by providing the infrastructure on which the SDN software can be run. In addition, the NFV is closely aligned with the SDN's objectives for the use of servers and switches.

\section{STATE OF THE ART}

The SDN and NFV technologies propose to revolutionize the way networks operate, and the success of these two technologies may well depend on their ability to interact harmoniously, if not support each other. To this end, and according to Ding, Qi, Wang, and Chen (2015), the SDN can provide connectivity between NFV files in a flexible and automated manner, thus simplifying network management. In addition, NFV can use the SDN as part of a service function chain (SFG). In this case, SDN controllers and business applications can run as NFV files in a scalable environment and benefit from essential features such as availability, reliability and elasticity. 
Several studies focus on the integration of NFV and SDN in different environments such as cloud computing, intelligent wide area networks, customer premises equipment, 5G, telemedicine, etc. These studies address several challenges, such as reliability, performance and scalability. These studies use a distinct architectural design logic as well as functional and non-functional requirements (Basta, Kellerer, Hoffmann, Morper, \& Hoffmann, 2014; Ordonez-Lucena, Ameigeiras, Lopez, Ramos-Munoz, Lorca, \& Folgueira, 2017; Vilalta et al., 2016).

Ensuring a good QoS level for multi-path networks is one of the major challenges, both for wireline networks $\mathrm{Wu}$, Cheng, Yuen, Cheung, \& Chen, 2015; Wu, Yuen, Cheng, Shang, \& Chen, 2014) and wireless networks more precisely those with energy consumption constraints (Wu, Cheng, Wang, \& Chen, 2018; Wu, Yuen, Cheng, Wang, \& Chen, 2016). The undesirable effects of multi-path and service degradation can only be truly felt when multimedia or real-time applications are routed through the network, more precisely and especially in wireless networks where bandwidth is one of the major concerns. Despite the many CMT (Concurrent Multipath Transfer) solutions, they remain limited due to the asymmetry of link performance and especially the sensitivity of some applications to SLA constraints. Wu, Yuen, Wang and Chen (2015) in their work considered as an improvement of CMT solutions considering video distortion in the path selection process, the proposed solution is published as Distortion Aware CMT (CMT-DA). The latter consists of first estimating the available bandwidth per path using Round Trip Time (RTT), congestion window and Timeout retransmission (RTO). Then, perform a flow rate allocation, i. e. send the acknowledgement packets via the most efficient uplinks in order to be able to adapt the congestion window. CMT-DA has been tested in a variety of heterogeneous wireless networks: WiFi, WiMax and cellular, the results obtained in terms of PSNR (Peak Signal to Noise Ratio), Goodput and InterPacket delay have shown the radical improvement in the QoS of multimedia traffic compared to existing wireless heterogeneous network solutions. 


\section{PROPOSED APPROACH}

Our approach is based on three logical layers; the service layer, the orchestration layer, and the physical layer. Figure 1 illustrates the architecture of the proposed approach.

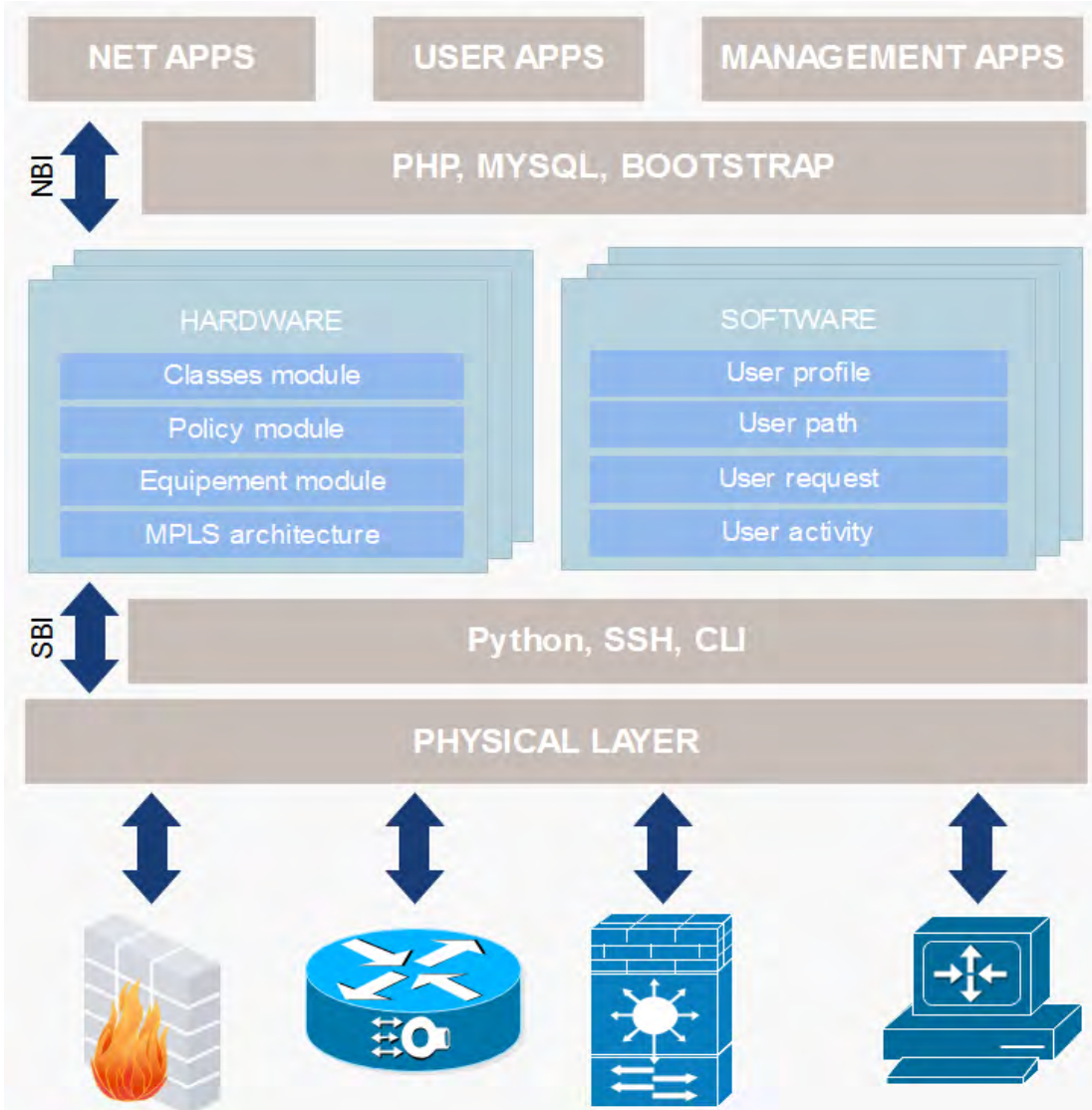

Figure 1. The proposed NFV architecture for virtualizing quality of service in an MPLS infrastructure.

The service layer provides all applications and services that affect the process of routing and routing MPLS frames (Bahnasse, Louhab, Oulahyane, Talea, \& Bakali, 2018). This layer provides all the graphical interfaces through which the infrastructure administrator can specify the applications to be used, their characteristics, and their QoS constraints. Figure 2 illustrates an example of the service layer interface. 


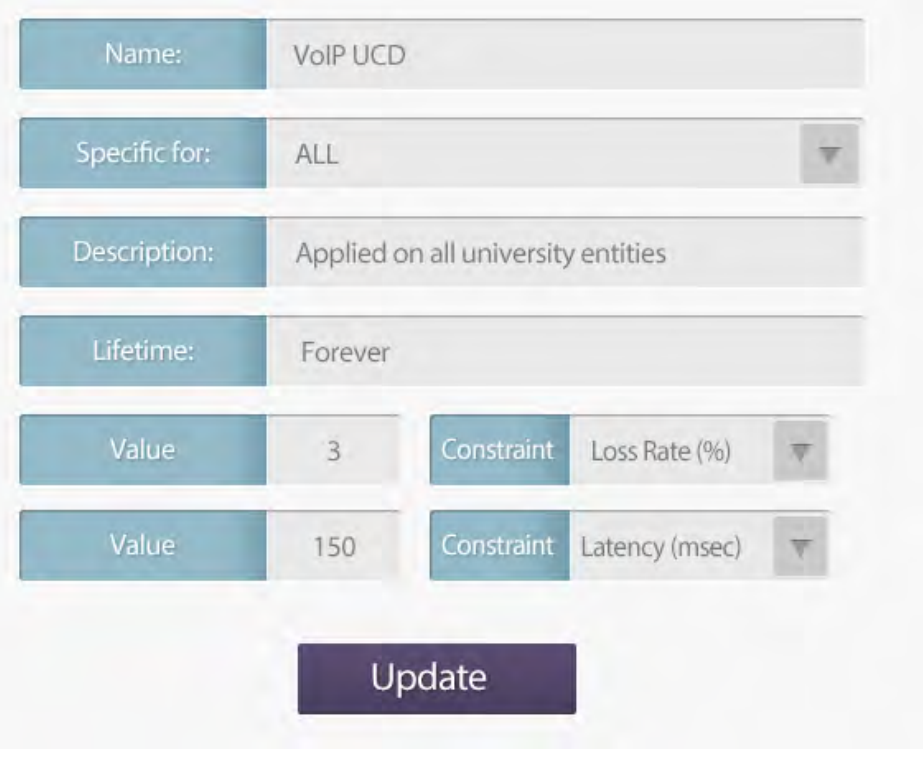

Figure 2. Service layer interface.

The software layer provides the necessary mechanisms for detecting user activity, including:

- The destination IP address;

- The state of the best way to this destination;

- The other possible paths for this destination;

- The user's subscription type;

- The bandwidth requested by the user;

- The user's permanent activity;

This layer is based on active metrology protocols (SNMP, CMIP) to ensure these objectives mentioned above.

The software layer also allows intelligent management of the paths that a user must take for a better quality of service. This path determination process is based on the RSVP protocol for the a priori establishment of the path. A list of paths is then 
defined in a stack sorted in terms of available bandwidth, the operating steps are as follows:

1. Calculate all paths between a source and a destination and store them in a stack.

2. Sort these paths according to the available bandwidth of each link.

3. Stack the results according to the number of links.

4. If the available bandwidth is enough to transmit user traffic, traffic is routed by the first path.

5. Otherwise, the priority of active traffic is checked. If it exceeds that of the generated traffic, then the traffic is routed by the following path.

To address the path with QoS constraints and the shortest path, we assume that the graph $\mathrm{G}=[\mathrm{X}, \mathrm{U}]$ represents a network of an $\mathrm{N}$ router and $\mathrm{M}$ links. Au, represents the available bandwidth of a link for each u $\mathrm{U}$.

for each request to establish an $\operatorname{LSP}(K)$, defined by a source $S(k)$, a destination $R(k)$, the requested bandwidth $\mathrm{dk}$ and the $\mathrm{nth}$ path between $\mathrm{s}(\mathrm{k})$ and $\mathrm{R}(\mathrm{k})$ for all $\mathrm{n}[1, \mathrm{P}(\mathrm{k})]$.

The links responding to bandwidth constraints are defined by:

$$
\operatorname{Min}\{\}, u[1, M]
$$

It is essential to detect a user's activity, and it is according to this activity that the routing process and QoS will be executed, to achieve this objective our model is based on the NBAR and Netflow protocols. Our model is based on flow sampling methods:

- Full: generates for each network flow an information that will be exported. This method is the oldest and the one supported on almost all routers but is no longer very common among operators because the router load and the amount of accounting information generated, especially during a shared denial of service, are too high. On the other hand, in the context of an internal network, 
it is almost mandatory if we want to be able to detect slow recognitions or violations of policies that try to be discreet.

- Sampled: Allows you to define the percentage of flows to be exported over the total number of flows generated. In general, operators are limited to 1 per 100 or even 1 per 1000. Even at 1 per 1000, a shared denial of service remains relatively easy to detect. The advantage of this method is the reduction of the router's CPU load and the amount of Netflow exported. The disadvantage is that it is not statistically good (deterministic function).

- Random Sampled: has been introduced relatively recently and on platforms of the 72xx/75xx type (whereas sampled was only available on GSR and 76xx, i.e. routers that support distributed CEF). The difference between sampled and random sampled is that the latter selects a random datagram from the configured $<x>$ which is statistically better.

Indeed, thanks to the monitoring of the user's activity, our model can detect the user's need in terms of bandwidth according to the type of application.

The Hardware layer allows several VRFs to be instantiated within the same gateway in accordance with user-specific routing policies. It allows to define within the gateways the different QoS classes and policies adapted for a user within a well determined architecture (MPLS, MPLS VPN, or traditional IP).

As soon as policies are configured, our platform establishes sockets with the different gateways of the network using the Python language. These sockets are used to connect to the gateways and execute information gathering and configuration commands, Figure 3 illustrates an example of the sockets used. 


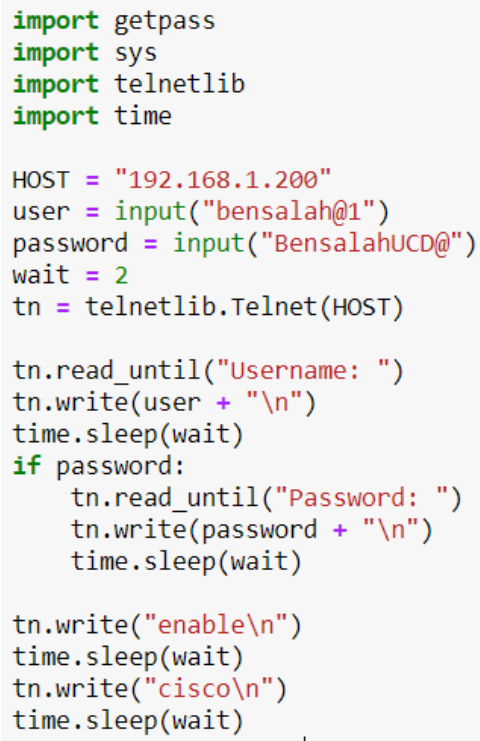

Figure 3. Python socket for connection with Cisco routers.

\section{CONCLUSIONS}

In this paper we have proposed a new architecture for virtualization of MPLS infrastructures by combining SDN and NFV approaches. The proposed architecture manages network services and equipment to meet subscriber QoS requirements. The adoption of such an architecture can provide unparalleled flexibility and cost reduction, as a single gateway can instantiate multiple virtual routing tables, each of which is subject to a routing policy that meets users' QoS requirements.

\section{REFERENCES}

Bahnasse, A., Louhab, F. E., Oulahyane, H. A., Talea, M., \& Bakali, A. (2018). Novel SDN architecture for smart MPLS traffic engineering-DiffServ aware management. Future Generation Computer Systems, 87, 115-126. doi: https:// doi.org/10.1016/j.future.2018.04.066 
Bahnasse, A., Louhab, F. E., Oulahyane, H. A., Talea, M., \& Bakali, A. (2018). Smart bandwidth allocation for next generation networks adopting software-defined network approach. Data in brief, 20, 840-845. doi: https://doi. org/10.1016/j.dib.2018.08.091

Basta, A., Kellerer, W., Hoffmann, M., Morper, H. J., \& Hoffmann, K. (2014, August). Applying NFV and SDN to LTE mobile core gateways, the functions placement problem. In Proceedings of the 4th workshop on All things cellular: operations, applications, \& challenges, 33-38. ACM. doi: https://doi. org/10.1145/2627585.2627592

Ding, W., Qi, W., Wang, J., \& Chen, B. (2015). OpenSCaaS: an open service chain as a service platform toward the integration of SDN and NFV. IEEE Network, 29(3), 30-35. doi: https://doi.org/10.1109/MNET.2015.7113222

Hawilo, H., Shami, A., Mirahmadi, M., \& Asal, R. (2014). NFV: State of the art, challenges and implementation in next generation mobile networks (vEPC). arXiv preprint arXiv:1409.4149

Ordonez-Lucena, J., Ameigeiras, P., Lopez, D., Ramos-Munoz, J. J., Lorca, J., \& Folgueira, J. (2017). Network slicing for 5G with SDN/NFV: Concepts, architectures, and challenges. IEEE Communications Magazine, 55(5), 80-87. doi: https://doi.org/10.1109/MCOM.2017.1600935

Vilalta, R., Mayoral, A., Pubill, D., Gasellas, R., Martínez, R., Serra, J., ... Muñoz, R. (2016, March). End-to-end SDN orchestration of IoT services using an SDN/NFV-enabled edge node. In 2016 Optical Fiber Communications Conference and Exhibition (OFC), 1-3. IEEE. doi: https://doi.org/10.1364/OFG.2016. W2A.42

Wu, J., Cheng, B., Wang, M., \& Chen, J. (2018). Energy-aware concurrent multipath transfer for real-time video streaming over heterogeneous wireless networks. IEEE Transactions on Circuits and Systems for Video Technology, 28(8), 20072023. doi: https://doi.org/10.1109/tcsvt.2017.2695368 
Wu, J., Gheng, B., Yuen, C., Cheung, N. M., \& Chen, J. (2015). Trading delay for distortion in one-way video communication over the internet. IEEE Transactions on Circuits and Systems for Video Technology, 26(4), 711-723. doi: https:// doi.org/10.1109/tcsvt.2015.2412774

Wu, J., Yuen, G., Gheng, B., Shang, Y., \& Ghen, J. (2014). Goodput-aware load distribution for real-time traffic over multipath networks. IEEE Transactions on Parallel and Distributed Systems, 26(8), 2286-2299. doi: https://doi.org/10.1109/ TPDS.2014.2347031

Wu, J., Yuen, C., Cheng, B., Wang, M., \& Chen, J. (2016). Energy-minimized multipath video transport to mobile devices in heterogeneous wireless networks. IEEE Journal on Selected Areas in Communications, 34(5), 1160-1178. doi: https: / doi. org/10.1109/JSAC.2016.2551483

Wu, J., Yuen, C., Wang, M., \& Ghen, J. (2015). Content-aware concurrent multipath transfer for high-definition video streaming over heterogeneous wireless networks. IEEE Transactions on Parallel and Distributed Systems, 27(3), 710-723. doi: https://doi.org/10.1109/TPDS.2015.2416736 
Edición Especial Special Issue Noviembre 2019

DOI: http://dx.doi.org/10.17993/3ctecno.2019.specialissue3.107-119 